\title{
Nutzen der Zentralisierung hochspezialisierter Leistungen in Frage gestellt
}

\author{
Bei der aktuellen Diskussion in der Schweiz über Spitallisten und die sog. hochspe- \\ zialisierte Medizin geht man von der Annahme aus, dass eine Konzentration von \\ Leistungen die Behandlungsqualität verbessert. Eine norwegische Studie lässt Zwei- \\ fel an dieser Beziehung aufkommen.
}

\author{
Adrian Merlo ${ }^{a}$ René \\ Bernays $^{b}$, Oliver Hausmann ${ }^{c}$, \\ Hans Landolt ${ }^{d}$, Michael \\ Reinert $^{e}$, Karl Schaller ${ }^{f}$ \\ a Prof. Dr. med., Past-Präsident \\ Schweizerische Gesellschaft \\ für Neurochirurgie (SGNC) \\ b Prof. Dr. med., Zürich, \\ Präsident SGNC \\ c PD Dr. med., Luzern, \\ Vorstand der SGNC \\ d Prof Dr. med., Aarau, \\ Vorstand der SGNC \\ e Prof Dr. med., Bern, \\ Vorstand der SGNC \\ f Prof. Dr. med., Genf, \\ Vorstand der SGNC
}

* Die Literaturangaben finden sich unter www.saez.ch $\rightarrow$ Aktuelle Numme oder $\rightarrow$ Archiv $\rightarrow 2012 \rightarrow 31 / 32$

Korrespondenz:

Prof. Dr. med. Adrian Merlo Kramgasse 30

CH-3011 Bern

adrian.merlo[at]gmx.ch
Im European Journal of Neurosurgery (Acta Neurochirurgica) wurde 2011 durch eine Studie aus Norwegen eine Grundsatzdebatte über den Nutzen der Zentralisierung hochspezialisierter medizinischer Leistungen lanciert [1] ${ }^{*}$, die den in Politik und Ärzteschaft verbreiteten Glauben in Frage stellt, dass sich durch Konzentration von Leistungen die Behandlungsqualität automatisch verbessern lässt. Der Diskurs wird zwischen Neurochirurgen aus Norwegen und pädiatrischen Neurochirurgen aus England geführt [2].

\section{Die Norwegische Studie}

Worum geht es? Die Grösse des Zuweiservolumens steht im Brennpunkt von Qualitätsdiskussionen. In Norwegen gibt es aufgrund der Populationsverteilung grössere Schwankungen beim Zuweiservolumen. Das Überleben von Kindern mit Hirntumoren wurde mit Hilfe des nationalen Krebsregisters über einen Zeitraum von 20 Jahren für alle Zentren des Landes erfasst. Für das Gesamttotal von 816 Patienten ergaben sich keine Unterschiede für die jeweiligen Zentren unabhängig von den Fallzahlen. Bezüglich der Subgruppe der Medulloblastome/PNET zeigte sich ein signifikant schlechteres Überleben im Zentrum mit dem höchsten Zuweiservolumen. Das Outcome bei diesem Kleinhirntumor ist direkt von der möglichst radikalen Resektion abhängig. Das Ergebnis der norwegischen Studie ist unerwartet und widerspricht dem gegenwärtig weitverbreiteten Imperativ, die Behandlungsqualität durch Zentralisierung von Leistungen zu optimieren. Wie interpretieren die norwegischen Autoren das unerwartete Resultat? Sie argumentieren, dass Hochvolumenzentren auch eine Vielzahl einfacherer Routinefälle anziehen, welche die Ärzte absorbieren. Zudem nimmt in grossen Zentren der administrative Aufwand pro Arzt massiv zu, so dass das spezifische chirurgische Training demjenigen an einem Niedrigvolumenzentrum nicht überlegen ist. Für die norwe- gischen Fachspezialisten ist die individuelle Kompetenz des Chirurgen der Schlüsselfaktor für das Behandlungsresultat, weitgehend unabhängig von der Fallzahl. Höhere Fallzahlen führen nicht zwingend zu besseren Ergebnissen.

\section{Schwache Datenlage internationaler Studien zur Volumen-Outcome-Beziehung}

In der Replik zu den Einwänden der englischen Kollegen [2] analysieren die norwegischen Autoren kritisch die gegenwärtige internationale Datenlage [3]. Fast alle Befürworter von Zentralisierung stützen sich auf Zahlen von amerikanischen Gesundheitsdatenbanken, die wenig klinisches Material enthalten. US-Zentren mit hohem Patientenvolumen behandeln jüngere, wohlhabendere, weisse Patienten mit gutem Versicherungsstatus, was zu einer hohen Zahl elektiver Fälle führt. Demgegenüber hat eine von einer grossen Versicherungsgesellschaft in Auftrag gegebene Outcome-Studie in US-amerikanischen Veterans Affair-Spitälern, bei der prospektiv klinische Daten in vielen chirurgischen Disziplinen gesammelt wurden, keine Korrelation zwischen Patientenvolumen und Risiko-adaptiertem Outcome herstellen können [4]. Was nun die neurochirurgischen Eingriffe anbelangt, gibt es offenbar weniger als ein Dutzend Publikationen, die eine positive Korrelation zwischen Zuweiservolumen und Outcome postulieren, wobei neun dieser Studien aus den USA und sechs davon vom selben Autor stammen. Eine kanadische [5] und die hier diskutierte norwegische Studie [1] stellen die inverse Korrelation zwischen $\mathrm{Zu}$ weiservolumen und Mortalität in Frage. Eine genaue Analyse von US-amerikanischen Daten - es handelte sich dabei um transphenoidale Hypophysenadenom-Operationen - zeigt aber, dass $64 \%$ der Chirurgen in Hochvolumenzentren nur 1-4 Fälle pro Jahr behandelten, ja dass sogar 55\% der Chirurgen nur einen Fall pro Jahr behandelt hatten. Bei den pädiat- 
rischen Hirntumoren behandelten in den USA 66\% der Neurochirurgen in Hochvolumenzentren nur einen Fall pro Jahr. Ähnlich sind die Zahlen für Meningeom- und Hydrozephalusoperationen oder für das Aneurysmaclipping. Daraus ergibt sich, dass die Definition eines bestimmten Volumens pro Zentrum resp. von spezifischen Fallzahlen pro Operateur aufgrund solchen Zahlenmaterials willkürlich erscheinen muss [3].

\section{«Der Wille zur Zentralisierung basiert nicht wirklich auf Evidenz, dazu sind die Daten zu widersprüchlich.»}

\section{Die Argumente der Zentralisierungs- befürworter}

Eine frühe Studie über das Verhältnis von Behandlungsvolumen und Mortalität hat bei einigen der untersuchten Eingriffe eine klare Reduktion der Mortalität bei grösserem Patientenvolumen gemessen, wobei die Autoren nicht zwischen chirurgischer Erfahrung, Zuweisungspraxis oder Patientenselektion differenzieren konnten. Dennoch wird aus den Daten der Schluss gezogen, dass die Zentralisierung von Leistungen anzustreben sei [6]. Eine weitere Studie aus jener Zeit, die nicht zitiert wird, hat denselben Zusammenhang zwischen Mortalität und Operationsvolumen gefunden, kam aber in der Detailanalyse zum Schluss, dass die höhere Mortalität nicht durch schlechtere Chirurgie, sondern durch Mängel des Spitals bedingt war [7]. Bei der Oesophagektomie scheint die Behandlungsmortalität vom Operationsvolumen abzuhängen. Eine neuere, ebenfalls nicht diskutierte Studie zeigt aber, dass die Oesophagektomie-Mortalität in Spitälern mit zum Teil sehr kleinem Volumen (1 Fall/Jahr!) niedrig sein kann, wenn die Pflegedichte hoch, die Ärzteschaft kompetent und das Spital technisch gut ausgerüstet ist. Schlechtere Behandlungsresultate gab es in vernachlässigten Inner City Hospitals, die sozial Benachteiligte behandeln [8].

Die Befürworter der Zentralisierung zitieren eine neuere australische Studie, bei der eine ausgedehnte Meta-Analyse durchgeführt wurde [9]. In dieser Meta-Analyse werden einige Verfahren mit negativer Korrelation zwischen Behandlungsvolumen und Mortalität aufgelistet. Die Autoren merken kritisch an, dass für eine geringe Senkung der Mortalität erhebliche Patientenverschiebungen notwendig wären, und dass das Fallvolumen wohl kein guter Parameter ist für die Messung der Behandlungsqualität. Eine neue US-amerikanische Studie über Patientenvolumen und Outcome bei der chirurgischen Behandlung von Spinalstenosen zeigt, dass bei sehr kleinen Fallzahlen (ein Fall pro Jahr) die Komplika- tionsrate höher ist [10]. Ob bei der nationalen Spitalstatistik, woraus die Zahlen extrahiert wurden, nach Ausrüstungsstand, Standort und Pflegedichte differenziert wurde, ist unklar. Bezüglich kindlicher Hirntumoren wird die kanadische Studie von 1996 zitiert, die auf Krebsregisterdaten des Staates Ontario beruht, die ein besseres 5-Jahres-Überleben bei Medulloblastomen gezeigt hat, wenn diese zentralisiert durchgeführt wurde [5]. Es handelt sich allerdings um Populationsdaten von 1977 bis 1987. Dabei stellte sich das Resttumorvolumen nach Resektion als der kritische prognostische Faktor heraus. Bei der multivariaten Analyse - nach Korrektur für Resektionsausmass, Tumorstadium, Geschlecht und Meningitis ergab sich keine Signifikanz zugunsten des Hochvolumenzentrums. Eine amerikanische Studie untersuchte den Zusammenhang zwischen Resektionsausmass und Trainingsgrad der Neurochirurgen bei malignen kindlichen Hirntumoren [11]. Dabei zeigte sich, dass der Resektionsgrad besser war bei subspezialisierten pädiatrischen Tumor-Neurochirurgen im Vergleich zu allgemeinen oder pädiatrischen Neurochirurgen.

\section{Schlussfolgerungen}

«Übung macht den Meister!» Dies ist unbestritten. Es scheint aber klar, dass chirurgische Kompetenz nicht direkt von der Fallzahl eines spezifischen Eingriffs abhängt. Vielmehr resultiert diese aus einer jahrelangen gezielten Aus- und Fortbildung, ähnlich wie bei anderen Berufen, bei denen sich manuelles Geschick, analytische Begabung und die Fähigkeit zu entschlossenem Handeln ineinander verweben zu einem komplexen Handlungsmuster. In der medizinischen Diskussion ist die Datenlage kontrovers. Der Wille zur Zentralisierung basiert nicht wirklich auf Evidenz, dazu sind die Daten zu widersprüchlich, sondern, wie auch die norwegischen Spezialisten betonen, auf einem persönlichen Glauben, der mit ökonomischen und politischen Argumenten untermauert wird. Bei der aktuellen Diskussion in der Schweiz über Spitallisten und die sogenannte hochspezialisierte Medizin (HSM) werden für zahlreiche Eingriffe willkürlich Mindestfallzahlen gesetzt. In einer Zeit der ökonomischen Definitionshoheit vieler Lebensbereiche dürfte es um Kostensenkung gehen. Es ist aber fraglich, ob ein Grossbetrieb mit komplexer Organisationsform die gleiche Leistung zum gleichen Preis anbieten kann wie ein gut ausgerüstetes kleineres Spital. Die norwegische Studie zeigt unerwartet, dass ein komplexeres Umfeld das Behandlungsresultat negativ beeinflussen kann.

Von regulatorischer Seite wird auch argumentiert, dass Zentralisierung notwendig sei, um genügend Fälle für die Ausbildung des chirurgischen Nachwuchses bereitzustellen. Die Schaffung von staatlichen Monopolen dürfte die Qualität jedoch kaum fördern. In Ländern mit freier Arztwahl kann der mündige Patient zwischen verschiedenen Spezia- 


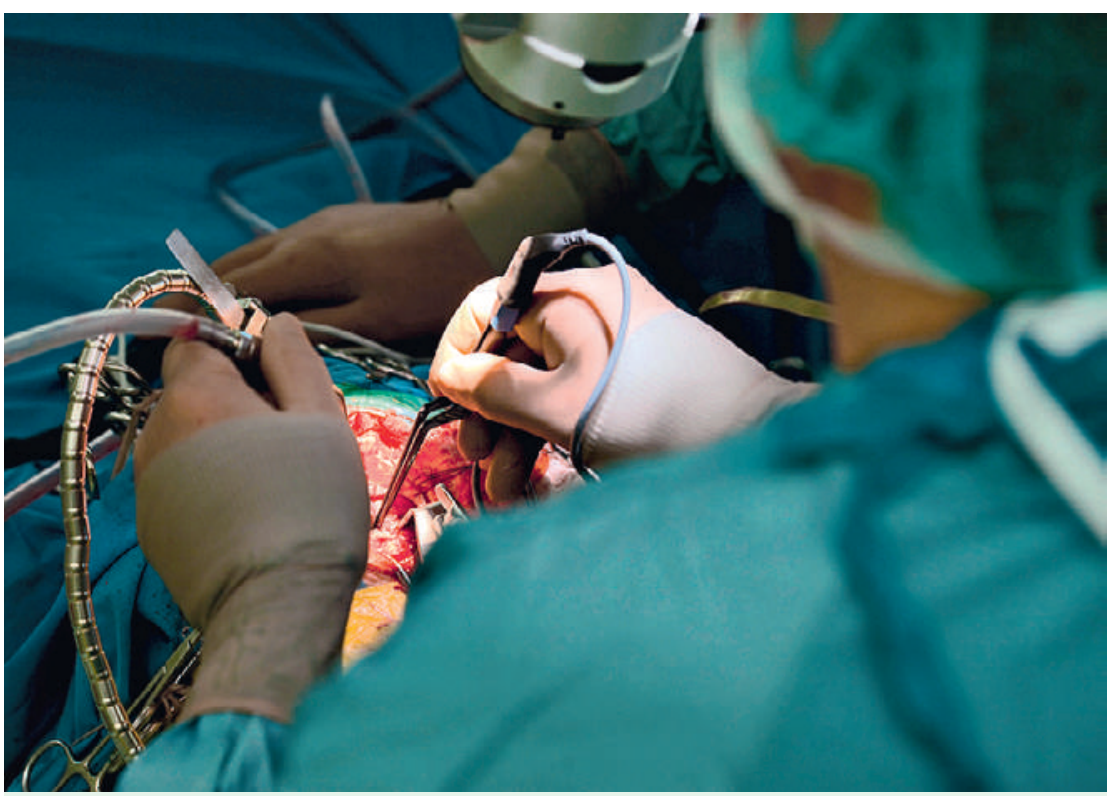

«Übung macht den Meister», dies ist unbestritten. Doch es scheint, dass chirurgische Kompetenz nicht direkt von der Fallzahl eines spezifischen Eingriffs abhängt.

listen und Spitälern auswählen, die miteinander im Wettbewerb stehen. Hochstehende Fachpflege und moderne Ausrüstung scheinen mindestens so wichtig zu sein wie das Behandlungsvolumen. In der Schweiz erscheint eine Zentralisierung von sogenannten HSM-Leistungen in jene grossen Spitäler,
Was müsste in der Schweiz wirklich verbessert werden? Antworten finden sich an der Front. Die Einführung der 50-Stunden-Woche hat in einigen Fächern zu einer Plethora von Ausbildungsstellen geführt. Die Anzahl Eingriffe pro Klinik ist aber kaum angestiegen. Das bedeutet, dass sich die endliche Zahl von Ausbildungsoperationen auf eine nahezu doppelt so grosse Zahl von Facharztanwärtern verteilt. Dies führt logischerweise entweder zu einer Verlängerung der Ausbildungszeit oder zu einer Verdünnung der chirurgischen Erfahrung, das heisst zu einer geringeren individuellen Kompetenz. Ein Zentrum mit höheren Fallzahlen und mehreren nicht sehr erfahrenen Operateuren dürfte einem kleineren Zentrum mit geringeren Fallzahlen und wenigen kompetenten Operateuren unterlegen sein. Die norwegische Studie dürfte diesen Sachverhalt reflektieren.

Wie aber kann die Qualität der Ausbildung gesichert werden? Erfahrene Chirurgen bilden einen begrenzten Kreis begabter Nachwuchschirurgen aus, was bedeutet, dass die Mehrzahl der Anwärter das ersehnte Ausbildungsziel nicht erreichen kann. Dieses Dilemma kann durch die Einführung von Subspezialisierungen abgeschwächt werden. Durch Subspezialisierung steigt der Bedarf an gutausgebildeten Spezialisten, gleichzeitig erhöht sich auch deren Kompetenz in ihrer Subspezialität. Ein solches Modell beruht auf Führungsverantwortung, Leistungsbereitschaft, Begabung und Wettbewerb. Um nicht eine grössere Zahl von mässig ausgebildeten Chirurgen

\section{«Hochstehende Fachpflege und moderne Ausrüstung scheinen mindestens so wichtig zu sein wie das Behandlungsvolumen.»}

die aufgrund jahrelanger einseitiger betriebswirtschaftlicher Umbauprozesse einen grossen Investitionsrückstand aufweisen, die Optimierung der Behandlungsqualität nicht zu garantieren. Stellt sich die Frage, ob nicht neue Formen der fachlichen Kooperation zwischen öffentlichen, aber auch zwischen öffentlichen und privaten Häusern für die Ausbildung ein Lösungsansatz ist. Bezüglich diverser spezialisierter Behandlungsverfahren haben die Kantone den Weg der willkürlichen Verteilung von Leistungen aufgrund von Fallzahlen eingeschlagen. Dabei gibt es dringendere Probleme anzupacken, wie z.B. die seit vielen Jahren insuffiziente Förderung des ärztlichen Nachwuchses. Stattdessen verordnet die Politik dem noch gut funktionierenden Gesundheitssystem teure betriebswirtschaftliche Revolutionen und eine Zulassungspraxis, die zwischen Totalstopp und Laisser-faire oszilliert. Wie ein zu stark geschnürtes Korsett könnte dies die Grund- und Spezialversorgung in die Asphyxie treiben. heranzuziehen, müssen die Strukturen in den Kliniken gestrafft und umgebaut werden. Für die Einhaltung der 50-Stunden-Woche braucht es im Fach Neurochirurgie auf den Nicht-Ausbildungsstellen Rotationsassistenten aus den Nachbarfächern (Neurologie, Neuroradiologie, Anästhesie, Intensivmedizin, Notfallmedizin, Psychiatrie usw.). Zudem muss die 50-Stunden-Woche für die eigentlichen Fachanwärter gelockert werden, damit der individuelle Erfahrungsschatz nicht strukturell verdünnt resp. die Ausbildung nicht ungebührlich verlängert wird. Die politisch Verantwortlichen täten gut daran, in $\mathrm{Zu}$ sammenarbeit mit den Fachgesellschaften die echten Qualitätsprobleme in der realen Ausbildungssituation anzugehen, anstatt mit unergiebigen Verteilungsschlüsseln aufgrund wenig aussagekräftiger Fallzahlen an den wirklichen Problemen der Front vorbeizudirigieren. 